\title{
What Do We Mean by Multicellularity? The Evolutionary Transitions Framework Provides Answers
}

\author{
Caroline J. Rose ${ }^{1 \dagger}$ and Katrin Hammerschmidt ${ }^{2 * \dagger}$ \\ ${ }^{1}$ Centre d'Écologie Fonctionnelle et Évolutive (CEFE), Centre National de la Recherche Scientifique (CNRS), Université de \\ Montpellier, Université Paul Valéry Montpellier, Montpellier, France, ${ }^{2}$ Institute of General Microbiology, Department of Biology, \\ Kiel University, Kiel, Germany
}

Keywords: multicellularity, Darwinian individuality, major evolutionary transitions, unit of selection, collective

\section{INTRODUCTION}

OPEN ACCESS

Edited by:

Peter Nonacs,

University of California, Los Angeles,

United States

Reviewed by:

Thibaut Brunet,

University of California, Berkeley,

United States

${ }^{*}$ Correspondence:

Katrin Hammerschmidt

katrinhammerschmidt@

googlemail.com

TORCID:

Caroline J. Rose

orcid.org/0000-0001-7133-0355

Katrin Hammerschmidt

orcid.org/0000-0003-0172-8995

Specialty section:

This article was submitted to

Social Evolution,

a section of the journal

Frontiers in Ecology and Evolution

Received: 25 June 2021

Accepted: 15 October 2021

Published: 19 November 2021

Citation:

Rose $\mathrm{CJ}$ and Hammerschmidt $\mathrm{K}$ (2021) What Do We Mean by Multicellularity? The Evolutionary Transitions Framework Provides Answers. Front. Ecol. Evol. 9:730714. doi: 10.3389/fevo.2021.730714
At first glance, the meaning of the word "multicellularity" appears to be unambiguous - it is treated as an "intuitive" concept, something that can be grasped with common sense. On closer inspection, however, it is apparent that there is notable disparity in the recent literature regarding the usage of the term "multicellularity." Whereas, traditionally it was mainly attributed to complex organisms (Grosberg and Strathmann, 2007), more recently it has also been used for simple microbial colonies or biofilms (Hengge, 2020). Accordingly, a unifying definition is lacking-whereas some definitions require cells to display an overall coordination of function (Wolpert and Szathmáry, 2002), have physical contact and strong interactions (Kaiser, 2001), others are simply based on the presence of a group-morphology (Schirrmeister et al., 2013).

We think that it is important to be more precise when using the term multicellularity as, for example, a microbial colony differs in important ways from a multicellular organism like us. This distinction has implications for various areas of inquiry such as "the sociobiology of microbes" and "the evolutionary transition to multicellularity." While these research directions have brought together a highly interdisciplinary community of researchers, adequate descriptions of the marginal or nascent cases of multicellularity remain elusive, despite their identification across the entire range of model organisms, such as algae, protozoans, yeast, and bacteria (Ratcliff et al., 2012; Claessen et al., 2014; Hammerschmidt et al., 2014; van Gestel and Tarnita, 2017; Brunet et al., 2019; Kapsetaki and West, 2019). Lack of continuity has also led to vastly different estimates of the number of instances of multicellular emergence in evolutionary history (Niklas and Newman, 2020). Depending on the definition of multicellularity, it is thought to have evolved from unicellular ancestors on 13-25 independent occasions. When described simply as a cellular aggregation, multicellular organisms are estimated conservatively to have evolved in at least 25 lineages (Grosberg and Strathmann, 2007), making it a "minor major" evolutionary transition. When more stringent criteria are applied, as for example a requirement for sustained cell-to-cell interconnection, communication, and cooperation, multicellularity has evolved multiple times in bacteria (e.g., Actinobacteria, Myxobacteria, and Cyanobacteria; see Bonner, 2000), but only once in the Animalia, three times in the Fungi (chytrids, ascomycetes, and basidiomycetes), and six times among the algae (twice each in the rhodophytes, stramenopiles, and chlorobionta; Niklas and Newman, 2013).

We argue that we need a better understanding about what multicellularity is to meaningfully discuss factors that determine its evolution. We propose that clarity can be achieved with the realization that the various definitions of multicellularity are in fact describing different stages that can occur during the course of its evolution. The major evolutionary transition from 
single cells to multicellular organisms is not an instantaneous shift, but rather a process with multiple transient stages. As such, "multicellularity" itself is not necessarily a fixed state, but exists as a large range encompassing single cells that are part of multicellular groups, multicellular individuals, and multicellular organisms. We here provide a framework for identifying the various stages of the transition to multicellularity. Importantly, we do not intend to imply that fixed boundaries separate stages of an evolutionary transition from single cells to multicellular organisms. We only demarcate stages here to provide a conceptual link between semantic use and a dynamic evolutionary process.

\section{THE EVOLUTION OF MULTICELLULARITY-A DYNAMIC PROCESS}

The transition to multicellularity begins with the evolution of cooperation, where cells unite together and gain an advantage over solitary cells (Stage One; Figure 1). The focus of natural selection remains on cells, albeit in a group-structured context. Stage Two is the true "transitional stage" of a major evolutionary transition, where the cooperating group also becomes a unit of selection-a "Darwinian individual." Crucially, in order to satisfy the conditions of Darwinian individuality, the groups themselves are subject to a process of reproduction and selection that is more than simply selection among their constituent cells (Godfrey-Smith, 2009). A high degree of functional organization is an adaptation of groups, resulting from selection operating at the higher (group) level (Okasha, 2006). Therefore, complex adaptations of groups accumulate during the third stage of an evolutionary transition. Eventually, group adaptations lead to such integration of the cells comprising the group that they can no longer exist independently, and now only survive and replicate as components of the multicellular group-the "organism" (Stage Three). In contrast to the view of Bourke (2011), who proposes that only complex multicellular organisms possess individuality, in our view individuality occurs at an earlier phase of the transition (Stage Two).

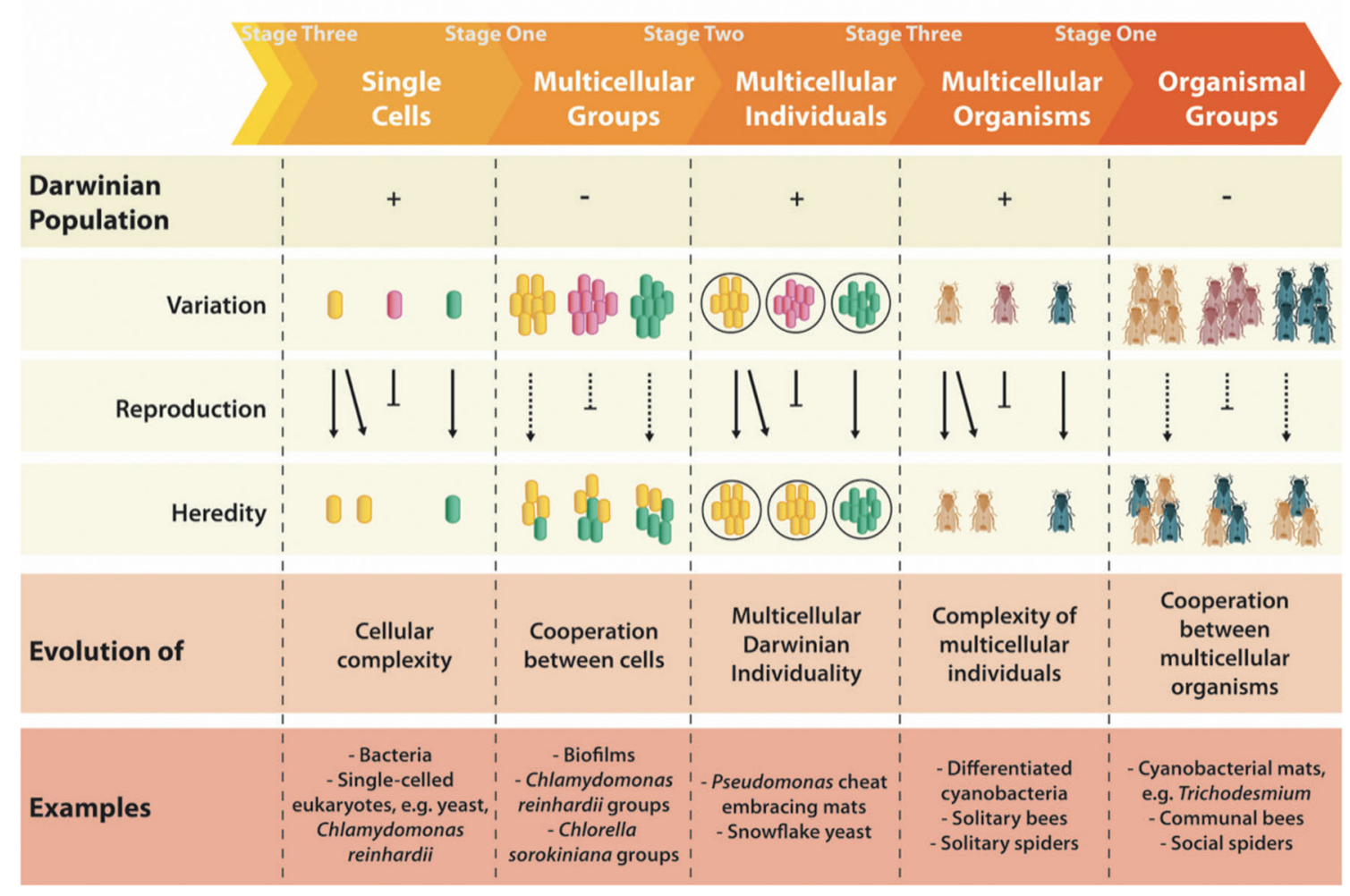

FIGURE 1 | Multicellular entities are defined as Multicellular Groups, Multicellular Individuals, or Multicellular Organisms, reflecting the stages of the evolutionary transition from single cells to multicellularity imbedded within the hierarchical structure of life. "Darwinian populations" are populations of "Darwinian individuals" or "units of selection," which are entities that can evolve by natural selection by virtue of possessing these essential characteristics: variation between entities within the population, which is heritable and causally associated with their differential reproduction (Lewontin, 1970). The (+) symbol signifies that, for example, single cells are units of selection in a Darwinian population (of cells). While multicellular groups may display a marginal kind of reproduction by virtue of differential growth (i.e., reproduction of cells), they do not meet other criteria for Darwinian individuality, such as (group) heredity, and therefore the (-) symbol indicates that multicellular groups are not members of a Darwinian population (of groups). Such criteria are the subject of much debate, and include both intrinsic features and ecological requirements (see Stage Two). Colors of different entities represent genetic differences; solid arrows and bars represent differential reproduction, while dotted arrows and bars represent differential growth of groups. 


\section{STAGE ONE-EVOLUTION OF MULTICELLULAR GROUPS}

The evolution of cooperation encapsulates Stage One of the evolution of multicellularity. A cooperative behavior is generally described as a costly investment in resources that benefits an individual (the recipient) other than the actor (Chase, 1980), regardless of whether the recipient adopts the same behavioral strategy. Cooperative interactions are central to an evolutionary transition because the necessary fitness cost associated with cooperation is offset by a grouplevel benefit.

During the transition to multicellularity, cooperation between cells resulted from the advantages gained by adhering to each other. This occurred through two mechanisms: clonality and aggregation (Grosberg and Strathmann, 2007; Tarnita et al., 2013). During a transition to clonal multicellularity, cells fail to adequately separate after cell division and ergo remain attached. Consequently, clonal forms of multicellularity, such as plants and animals (Stage Three), developed from a small number of cells (Stage One/Two) - an evolutionary "bottleneck." The aggregative mode of multicellularity usually results from motile single cells (Stage One/Two) clustering together to form fruiting bodies for sporulation and dispersal, often in response to environmental starvation (Gross, 1994). Aggregative forms of multicellularity have arisen independently in eubacteria, several cellular slime molds, and in ciliates (Bonner, 1998). While aggregative forms of multicellularity are numerous and widespread, particularly in terrestrial environments, clonal multicellularity has led to greater diversity and complexity (Fisher et al., 2013).

The challenge for understanding the evolution of cooperation is explaining how cooperation generates a benefit (Calcott, 2011). Multicellular cooperation in many lineages may have originally obtained the advantage of increased size afforded by the ever-present open niche at the top of the size scale (Bonner, 1998, 2000). Proposed advantages of increased size are that larger assemblages of cells avoid predation by filter feeders or that increased size enhances feeding efficiency (Dworkin, 1972; Bell, 1985; Bonner, 1998; Boraas et al., 1998; Pfeiffer et al., 2001; Alegado et al., 2012; Koschwanez et al., 2013; Herron et al., 2019; Kapsetaki and West, 2019). Other advantages of cellular cooperation include benefits associated with both fixed surface attachment and enhanced dispersal. Single cells located in an ideal position for growth may be swept away by currents or wind, whereas an increased ability to adhere to surfaces by cell clusters might be selectively advantageous (Gross, 1994; Bonner, 1998). Tradeoffs between two incompatible processes that cannot be performed in one cell at the same time have also been proposed as important drivers of multicellular cooperation. Examples of such tradeoffs include motility and mitosis in metazoans (Margulis, 1981; Buss, 1987; King, 2004), reproduction and motility in the volvocene green algae (Koufopanou, 1994), and $\mathrm{N}_{2}$ and $\mathrm{CO}_{2}$ fixation in cyanobacteria (Rossetti et al., 2010; Herrero et al., 2016; Hammerschmidt et al., 2021).

\section{STAGE TWO-EVOLUTION OF MULTICELLULAR INDIVIDUALS}

Stage Two is the true "transitional" phase of a major evolutionary transition because during this stage, natural selection operates between groups, rendering them "Darwinian individuals." In order to be a "unit of selection" (Lewontin, 1970), a group itself must become capable of a form of reproduction that allows selection to operate on the variation between groups, over and above selection already occurring between cells (Figure 1). The particular question of relevance to major evolutionary transitions is the puzzle of group reproducers (Godfrey-Smith, 2009)reproducing units comprised of particles which themselves have the capacity to reproduce. Multicellular groups, for example biofilms or Chlamydomonas reinhardtii groups (Herron et al., 2019), multicellular individuals, such as snowflake yeast (Ratcliff et al., 2012) or Pseudomonas cheat embracing mats (Hammerschmidt et al., 2014), and multicellular organisms (filamentous cyanobacteria, solitary bees) are all examples of group reproducers. The difficulty is to identify which are cases of reproduction of groups, and which are cases of growth of groups resulting from reproduction and structural organization of their particles (see Figure 1 for details).

This challenge is related to the problem of explaining how groups acquired a fundamental requirement for reproduction - a life cycle. The particular mode by which the earliest multicellular groups reproduce, for example through a dedicated (germ) cell or by fragmentation, has implications for their ability to transition in individuality and participate in natural selection (Ratcliff et al., 2012; Hammerschmidt et al., 2014). Furthermore, during this transitional phase, ecological conditions are of critical importance (Pichugin et al., 2019; Staps et al., 2019), such as structured environments that maintain the discreteness of groups, and crucially, their reproductive cells (Rose et al., 2020). Such conditions provide the ecological scaffold for selection to act on less-integrated groups until they complete the transition to "multicellular individuals" (Black et al., 2020). The challenge of identifying criteria for Darwinian individuality has been the subject of much recent discussion. Our aim here is not to review the mechanisms put forward to explain the transition to Darwinian individuality (e.g., Michod, 2005; Godfrey-Smith, 2009; Bourke, 2011; Hammerschmidt et al., 2014; Black et al., 2020; Rose et al., 2020; Bourrat et al., 2021), but rather to remove linguistic ambiguities that may impede fruitful debate.

\section{STAGE THREE-EVOLUTION OF MULTICELLULAR ORGANISMS}

After a multicellular group becomes a Darwinian individual, it is possible for natural selection to operate on traits that enhance the fitness of the group as a collective unit. The accumulation of such traits leads to the evolution of progressively higher complexity. Hence, the term "complexity" does not refer to a specific state reached by a multicellular organism, but it is a relative term used to describe a wide spectrum of collective functions. 
Multicellular complexity is often represented by the number of different cell types coexisting in the collective, although epigenetic control of this cellular differentiation is clearly an important innovation resulting from group-level selection (Buss, 1987; Arnellos et al., 2013). Epigenetic regulation of development itself evolves as increasingly more complex genetic networks. The accumulation of group adaptations may eventually lead to such a degree of integration of parts that the cells no longer exist independently - their survival and reproduction depends entirely on the survival of the group. We suggest that this loss of lower level autonomy be the defining feature of the term "organism," ultimately rendering an organism indivisible. In the level above multicellular organisms, eusocial insect colonies are sometimes referred to as "superorganisms" when the lower level units no longer exist autonomously and instead subsist as sterile workers. This has also been shown to involve an increase in complexity of gene networks (Kapheim et al., 2015).

The evolution of developmental regulation is mechanistically unproblematic because the genetic machinery for coordination of differentiated cell types existed in primitive "multicellular" prokaryotes and close eukaryotic unicellular relatives of metazoans (Gombar et al., 2014; Glöckner et al., 2016; SebéPedrós et al., 2016; Brunet and King, 2017). It is therefore surmised that few mutational steps should be required in a regulatory pathway to produce additional cellular differentiation. Indeed, thousands of differences in gene expression between cell types in multicellular organisms are often controlled by a small set of regulatory proteins. This is supported by the fact that the presence of many genes underlying multicellular development and function has been inferred in the unicellular ancestors of metazoans, algae, and fungi, providing strong indications that regulatory changes indeed led to the co-option of the ancestral genes (Hanschen et al., 2016; Sebé-Pedrós et al., 2016; Kiss et al., 2019). Nevertheless, important metazoan developmental gene families, notably the Hox genes, are not present in unicellular ancestors (Ruiz-Trillo et al., 2007), indicating that these gene regulatory pathways evolved later as a consequence of multicellular individuality.

\section{CONCLUSION}

The transition to multicellularity is of seminal biological significance as it led to the vast biological complexity and diversity we see on our planet today. Reconstructing the stages that occurred during the process of evolutionary transitions that took place in the distant past is a major challenge (Maynard

\section{REFERENCES}

Alegado, R. A., Brown, L. W., Cao, S., Dermenjian, R. K., Zuzow, R., Fairclough, S. R., et al. (2012). A bacterial sulfonolipid triggers multicellular development in the closest living relatives of animals. eLife 1:e00013. doi: 10.7554/eLife.00013.030

Arnellos, A., Moreno, A., and Ruiz-Mirazo, K. (2013). Organizational requirements for multicellular autonomy: insights from a comparative case study. Biol. Philos. 29, 851-884. doi: 10.1007/s10539-013-9387-x
Smith and Szathmáry, 1995). While most research has focused on theoretical and philosophical aspects of these events, several recent developments and novel techniques have transformed this research area and brought together a highly interdisciplinary community of researchers who are rapidly advancing the field. One novel approach has been the utilization of unicellular model organisms, such as yeast, algae, protozoans, and bacteria in experimental evolution studies to mimic the evolution of early stages of the transition to multicellularity (Ratcliff et al., 2012; Claessen et al., 2014; Hammerschmidt et al., 2014; van Gestel and Tarnita, 2017; Brunet et al., 2019; Kapsetaki and West, 2019).

This new research direction has already contributed many exciting results that feed back into theory. However, these studies have also led to confusion regarding the definition of the term "multicellular," because they focus on marginal or nascent cases of multicellularity. In addition, the utility of the various definitions of multicellularity remains vague for extant organisms (Kaiser, 2001; Wolpert and Szathmáry, 2002; Grosberg and Strathmann, 2007; Schirrmeister et al., 2013; Hengge, 2020). We advocate that clarity can be achieved by considering the diverse use of the term "multicellularity" as sequential stages of a dynamic evolutionary process, from multicellular groups, to multicellular individuals, and finally to multicellular organisms. Semantic continuity among researchers will lead to more productive communication between evolutionary biologists and ecologists, microbiologists, philosophers, physicists and theoreticians, further advancing this exciting field.

\section{AUTHOR CONTRIBUTIONS}

Both authors contributed to the article and approved the submitted version.

\section{FUNDING}

$\mathrm{KH}$ thanks the HIAS (Hamburg Institute for Advanced Study) and the Joachim Herz Foundation for support. We acknowledge financial support by the DFG within the funding programme Open Access Publishing.

\section{ACKNOWLEDGMENTS}

We thank Pierrick Bourrat, Peter Deines, Peter Nonacs, Thibaut Brunet, and Walter Veit for comments on the manuscript. Figure created with Adobe Illustrator CS5.1. illustrated in the Volvocales," in The Origin and Evolution of Sex, eds H. Halvorson and A. Monroy (New York, NY: Alan R. Liss), 221-256.

Black, A. J., Bourrat, P., and Rainey, P. B. (2020). Ecological scaffolding and the evolution of individuality. Nat. Ecol. Evol. 4, 426-436. doi: 10.1038/s41559-019-1086-9

Bonner, J. T. (1998). The origins of multicellularity. Integr. Biol. 1, 27-36. doi: 10. 1002/(SICI)1520-6602(1998)1:1<27::AID-INBI4>3.0.CO;2-6 
Bonner, J. T. (2000). First Signals: The Evolution of Multicellular Development. Princeton, NJ: Princeton University Press. doi: 10.1515/978140083 0589

Boraas, M., Seale, D., and Boxhorn, J. (1998). Phagotrophy by a flagellate selects for colonial prey: a possible origin of multicellularity. Evol. Ecol. 12, 153-64. doi: 10.1023/A:1006527528063

Bourke, A. F. (2011). Principles of Social Evolution. Oxford: Oxford University Press. doi: 10.1093/acprof:oso/9780199231157.001. 0001

Bourrat, P., Doulcier, G., Rose, C. J., Rainey, P. B., and Hammerschmidt, K. (2021). Beyond fitness decoupling: tradeoff-breaking during evolutionary transitions in individuality. bioRxiv [Preprint]. doi: 10.1101/2021.09.01.458526

Brunet, T., and King, N. (2017). The origin of animal multicellularity and cell differentiation. Dev. Cell 43, 124-140. doi: 10.1016/j.devcel.2017. 09.016

Brunet, T., Larson, B. T., Linden, T. A., Vermeij, M. J., McDonald, K., and King, N. (2019). Light-regulated collective contractility in a multicellular choanoflagellate. Science 366, 326-334. doi: 10.1126/science.aay2346

Buss, L. W. (1987). The Evolution of Individuality. Princeton, NJ: Princeton University Press.

Calcott, B. (2011). "Alternative patterns of explanation for major transitions," in The Major Transitions in Evolution Revisited, eds B. Calcott and K. Sterelny (Cambridge, MA: MIT Press), 35-51. doi: $10.7551 /$ mitpress/9780262015240.003.0003

Chase, I. D. (1980). Cooperative and noncooperative behavior in animals. Am. Nat. 115, 827-57. doi: 10.1086/283603

Claessen, D., Rozen, D. E., Kuipers, O. P., Søgaard-Andersen, L., and van Wezel, G. P. (2014). Bacterial solutions to multicellularity: a tale of biofilms, filaments and fruiting bodies. Nat. Rev. Microbiol. 12, 115-124. doi: 10.1038/nrmicro 3178

Dworkin, M. (1972). The myxobacteria: new directions in studies of prokaryotic development. Crit. Rev. Microbiol. 1, 435-52. doi: 10.3109/1040841720910 3873

Fisher, R. M., Cornwallis, C. K., and West, S. A. (2013). Group formation, relatedness, and the evolution of multicellularity. Curr. Biol. 23, 1120-25. doi: 10.1016/j.cub.2013.05.004

Glöckner, G., Lawal, H. M., Felder, M., Singh, R., Singer, G., Weijer, C. J., et al. (2016). The multicellularity genes of dictyostelid social amoebas. Nat. Comm. 7:12085. doi: $10.1038 /$ ncomms 12085

Godfrey-Smith, P. (2009). Darwinian Populations and Natural Selection. Oxford: Oxford University Press. doi: 10.1093/acprof:osobl/9780199552047.001. 0001

Gombar, S., MacCarthy, T., and Bergman, A. (2014). Epigenetics decouples mutational from environmental robustness. Did it also facilitate multicellularity? PLoS Comput. Biol. 10:e1003450. doi: 10.1371/journal.pcbi.1003450

Grosberg, R. K., and Strathmann, R. R. (2007). The evolution of multicellularity: a minor major transition? Annu. Rev. Ecol. Evol. Syst. 38, 621-654. doi: 10.1146/annurev.ecolsys.36.102403.114735

Gross, J. D. (1994). Developmental decisions in Dictyostelium discoideum. Microbiol. Rev. 58, 330-351. doi: 10.1128/mr.58.3.330-351. 1994

Hammerschmidt, K., Landan, G., Domingues Kümmel Tria, F., Alcorta, J., and Dagan, T. (2021). The order of trait emergence in the evolution of cyanobacterial multicellularity. GBE 13:evaa249. doi: 10.1093/gbe/ evaa249

Hammerschmidt, K., Rose, C. J., Kerr, B., and Rainey, P. B. (2014). Life cycles, fitness decoupling and the evolution of multicellularity. Nature 515, 75-79. doi: 10.1038/nature13884

Hanschen, E. R., Marriage, T. N., Ferris, P. J., Hamaji, T., Toyoda, A., Fujiyama, A., et al. (2016). The Gonium pectorale genome demonstrates co-option of cell cycle regulation during the evolution of multicellularity. Nat Comm. 7:11370. doi: $10.1038 /$ ncomms 11370

Hengge, R. (2020). Linking bacterial growth, survival, and multicellularity - small signaling molecules as triggers and drivers. Curr. Opin. Microbiol. 55, 57-66. doi: 10.1016/j.mib.2020.02.007
Herrero, A., Stavans, J., and Flores, E. (2016). The multicellular nature of filamentous heterocyst-forming cyanobacteria. FEMS Microbiol. Rev. 40, 831-854. doi: 10.1093/femsre/fuw029

Herron, M. D., Borin, J. M., Boswell, J. C., Walker, J., Chen, I. C. K., Knox, C. A., et al. (2019). De novo origins of multicellularity in response to predation. Sci. Rep. 9:2328. doi: 10.1038/s41598-019-39558-8

Kaiser, D. (2001). Building a multicellular organism. Ann. Rev. Genet. 35, 103-23. doi: 10.1146/annurev.genet.35.102401.090145

Kapheim, K. M., Pan, H., Li, C., Salzberg, S. L., Puiu, D., Magoc, T., et al. (2015). Genomic signatures of evolutionary transitions from solitary to group living. Science 348, 1139-1143. doi: 10.1126/science.aaa4788

Kapsetaki, S. E., and West, S. A. (2019). The costs and benefits of multicellular group formation in algae. Evolution 73,1296-1308. doi: 10.1111/evo. 13712

King, N. (2004). The unicellular ancestry of animal development. Dev. Cell 7, 313-25. doi: 10.1016/j.devcel.2004.08.010

Kiss, E., Hegedüs, B., Virágh, M., Varga, T., Merényi, Z., Kósz,ó, T., et al. (2019). Comparative genomics reveals the origin of fungal hyphae and multicellularity. Nat. Comm. 10:4080. doi: 10.1038/s41467-019-12085-w

Koschwanez, J. H., Foster, K. R., and Murray, A. W. (2013). Improved use of a public good selects for the evolution of undifferentiated multicellularity. eLife 2:e00367. doi: 10.7554/eLife.00367.028

Koufopanou, V. (1994). The evolution of soma in the volvocales. Am. Nat. 143, 907-931. doi: 10.1086/285639

Lewontin, R. C. (1970). The units of selection. Annu. Rev. Ecol. Syst. 1, 1-18. doi: 10.1146/annurev.es.01.110170.000245

Margulis, L. (1981). Symbiosis in Cell Evolution. San Francisco, CA: Freeman.

Maynard Smith, J., and Szathmáry, E. (1995). The Major Transitions in Evolution. Oxford: Oxford University Press.

Michod, R. E. (2005). On the transfer of fitness from the cell to the multicellular organism. Biol. Philos. 20, 967-987. doi: 10.1007/s10539-005-9018-2

Niklas, K. J., and Newman, S. A. (2013). The origins of multicellular organisms. Evol. Dev. 15, 41-52. doi: 10.1111/ede.12013

Niklas, K. J., and Newman, S. A. (2020). The many roads to and from multicellularity. J. Exp. Bot. 71, 3247-3253. doi: 10.1093/jxb/erz547

Okasha, S. (2006). Evolution and the Levels Of Selection. Oxford: Oxford University Press. doi: 10.1093/acprof:oso/9780199267972.001.0001

Pfeiffer, T., Schuster, S., and Bonhoeffer, S. (2001). Cooperation and competition in the evolution of ATP-producing pathways. Science 292, 504-507. doi: $10.1126 /$ science. 1058079

Pichugin, Y., Park, H. J., and Traulsen, A. (2019). Evolution of simple multicellular life cycles in dynamic environments. J. R. Soc. Interface 16:20190054. doi: 10.1098/rsif.2019.0054

Ratcliff, W. C., Denison, R. F., Borrello, M., and Travisano, M. (2012). Experimental evolution of multicellularity. Proc. Natl. Acad. Sci. U.S.A. 109, 1595-1600. doi: 10.1073/pnas.1115323109

Rose, C. J., Hammerschmidt, K., Pichugin, Y., and Rainey, P. B. (2020). Metapopulation structure and the evolutionary transition to multicellularity. Ecol. Lett. 23, 1380-1390. doi: 10.1111/ele.13570

Rossetti, V., Schirrmeister, B. E., Bernasconi, M. V., and Bagheri, H. C. (2010). The evolutionary path to terminal differentiation and division of labor in cyanobacteria. J. Theor. Biol. 262, 23-34. doi: 10.1016/j.jtbi.2009. 09.009

Ruiz-Trillo, I., Burger, G., Holland, P. W. H., King, N., Lang, B. F., Roger, A. J., et al. (2007). The origins of multicellularity: a multi-taxon genome initiative. Trends Genet. 23, 113-118. doi: 10.1016/j.tig.2007.01.005

Schirrmeister, B. E., de Vos, J. M., Antonelli, A., and Bagheri, H. C. (2013). Evolution of multicellularity coincided with increased diversification of cyanobacteria and the Great Oxidation Event. Proc. Natl. Acad. Sci. U.S.A. 110, 1791-1796. doi: 10.1073/pnas.1209927110

Sebé-Pedrós, A., Ballaré, C., Parra-Acero, H., Chiva, C., Tena, J. J., Sabidó, E., et al. (2016). The dynamic regulatory genome of Capsaspora and the origin of animal multicellularity. Cell 165, 1224-1237. doi: 10.1016/j.cell.2016.03.034

Staps, M., van Gestel, J., and Tarnita, C. E. (2019). Emergence of diverse life cycles and life histories at the origin of multicellularity. Nat. Ecol. Evol. 3, 1197-1205. doi: 10.1038/s41559-019-0940-0 
Tarnita, C. E., Taubes, C. H., and Nowak, M. A. (2013). Evolutionary construction by staying together and coming together. J. Theoret. Biol. 320, 10-22. doi: $10.1016 /$ j.jtbi.2012.11.022

van Gestel, J., and Tarnita, C. E. (2017). On the origin of biological construction, with a focus on multicellularity. Proc. Natl. Acad. Sci. U.S.A. 114, 11018-11026. doi: 10.1073/pnas.1704631114

Wolpert, L., and Szathmáry, E. (2002). Evolution and the egg. Nature 420:745. doi: $10.1038 / 420745 a$

Conflict of Interest: The authors declare that the research was conducted in the absence of any commercial or financial relationships that could be construed as a potential conflict of interest.
Publisher's Note: All claims expressed in this article are solely those of the authors and do not necessarily represent those of their affiliated organizations, or those of the publisher, the editors and the reviewers. Any product that may be evaluated in this article, or claim that may be made by its manufacturer, is not guaranteed or endorsed by the publisher.

Copyright (C) 2021 Rose and Hammerschmidt. This is an open-access article distributed under the terms of the Creative Commons Attribution License (CC BY). The use, distribution or reproduction in other forums is permitted, provided the original author(s) and the copyright owner(s) are credited and that the original publication in this journal is cited, in accordance with accepted academic practice. No use, distribution or reproduction is permitted which does not comply with these terms. 